\title{
NON-LINEAR THEORY OF CURVED ELASTIC SHEETS*
}

\author{
BY \\ E. BROMBERG AND J. J. STOKER \\ New' York University
}

1. Theories of plane and curved shells which neglect bending. ${ }^{1}$ The problem to be treated here is that of determining, under certain conditions to be stated later, the stresses and strains in a thin curved elastic sheet in the form of a surface of revolution held fixed at the edges and subjected to a uniform pressure normal to the sheet. The problem thus falls under the general class of problems treated in the theory of elastic shells.

In order to obtain a theory of elastic shells which is manageable from the mathematical point of view, it is customary to make assumptions ${ }^{2}$ of various kinds, in addition to those of the general theory of elasticity. These additional assumptions are usually based on the hypothesis that the shell is very thin. In what follows we shall be interested in theories which result when the following assumptions are made:

1) The strains due to the normal stress on elements parallel to the surface of the shell are small enough to be neglected safely.

2) All stresses are constant over the thickness of the shell.

The first assumption is almost always made by writers on the subject of thin shells. The second assumption of course rules out what are usually called bending stresses.

A linear theory of shells, with a considerable number of practical applications, has been worked out on the basis of the above two assumptions. It is usually referred to as the membrane theory of shells. ${ }^{3}$ The salient feature of the theory is that it is "statically determinate" since the stresses can be obtained from the equilibrium conditions alone without reference to the elastic deformations. This results in a very great simplification, by comparison with theories which do not neglect bending. However, the simplification is coupled with at least one rather serious disadvantage: it turns out that it is not possible to satisfy the kind of boundary conditions which it would be natural to impose in these problems, since the order of the system of differential equations is too low. For example, the condition of a fixed edge (that is, the condition requiring the displacements at the boundary to vanish) cannot be satisfied in general.

Most writers on the membrane theory of shells attribute the difficulty regarding

* Received May 1, 1945.

1 The theory developed in this paper is an outgrowth of a research project carried out by the College of Engineering of New York University, under a contract with the War Production Board. The investigation, which was largely experimental in character, was concerned with the feasibility of constructing buildings circular in form with a thin steel roof supported by excess air pressure on the inside of the building. The design problems which arose led to the theory presented here. In this case the sheets considered were so thin that there was no doubt about the validity of neglecting bending stresses.

2 For an exhaustive classification of the very numerous possibilities here, see the recent paper of Chien [3]. (Here and in what follows, numbers in square brackets refer to the bibliography at the end of the paper.)

${ }^{3}$ For full treatments of this theory and references to the literature, see the books of Flügge [5] and Timoshenko [12]. 
boundary conditions to the fact that bending is neglected, and it is true that use of the linear bending theory does make it possible to impose physically reasonable boundary conditions. However, there are cases in which the shells are so thin that the bending stresses are small compared with the "membrane" stresses. ${ }^{4}$ It seems not to have been noticed that a theory which neglects bending stresses, but which nevertheless makes it possible to satisfy physically reasonable boundary conditions, can be obtained by taking account of certain non-linear terms in the relations for the strains as functions of the displacements. This paper has as its main purpose the development of such a non-linear theory.

Our theory is a generalization of an already existing non-linear theory for the case of a plane sheet ${ }^{5}$ supported in some way at its boundary and subjected to normal pressure $p$. It is useful for our purposes to discuss the theory of plane sheets from a num-
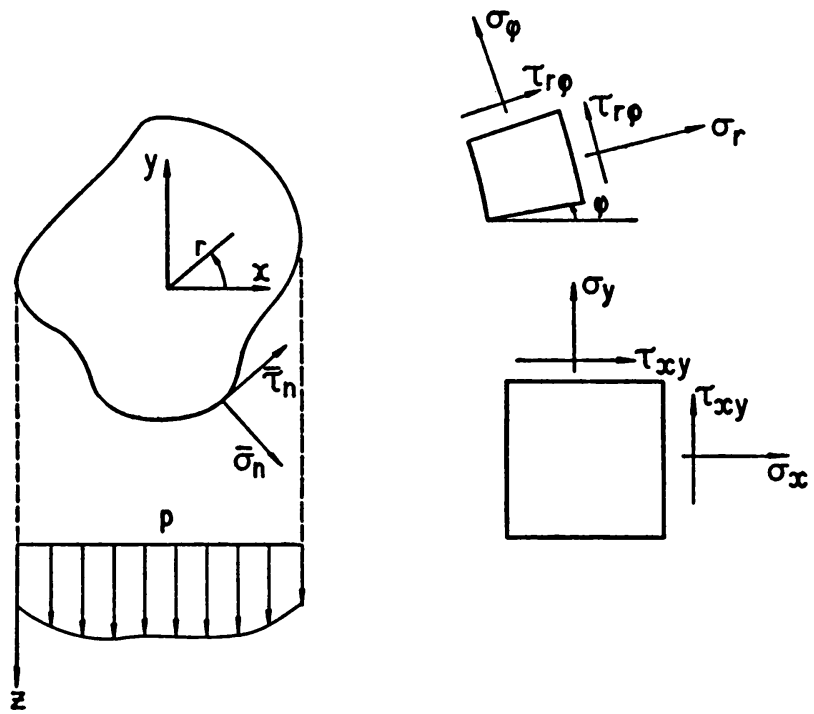

FIG. 1.

ber of different points of view, with the object of comparing and contrasting this theory with the theory of curved sheets to be presented later. The undeformed position of the sheet is taken as the $x y$-plane, the system of stresses in the sheet is denoted by $\sigma_{x}, \sigma_{y}$, and $\tau_{x y}$ and the displacement components by $u$, $v$, and $w$. In Fig. 1 the notation for the stresses $\sigma_{r}$ and $\sigma_{\phi}$ in polar coordinates $(r, \phi)$ is also indicated.

The equilibrium conditions for the stresses $\sigma_{x}, \sigma_{y}$, and $\tau_{x y}$ in the sheet are

$$
\frac{\partial \sigma_{x}}{\partial x}+\frac{\partial \tau_{x y}}{\partial y}=0, \quad \frac{\partial \tau_{x y}}{\partial x}+\frac{\partial \sigma_{y}}{\partial y}=0 .
$$

The equation of equilibrium for the direction normal to the sheet is

$$
\sigma_{y} \frac{\partial^{2} w}{\partial x^{2}}+2 \tau_{x y} \frac{\partial^{2} w}{\partial x \partial y}+\sigma_{x} \frac{\partial^{2} w}{\partial y^{2}}=-p / h
$$

4 The present investigation was prompted by the necessity of dealing with just such a case.

"The word "sheet" is employed here in a noncommittal way. In the course of our discussion a more precise significance will be given to the phrase "theory of thin sheets." 
where $h$ is the thickness of the sheet and $p$ is the pressure. The non-linear character of the theory under discussion stems from the retention of certain quadratic terms in the relations for the strains $\epsilon_{x}, \epsilon_{y}, \gamma_{x y}$ in terms of the displacements: ${ }^{6}$

$$
\begin{gathered}
\epsilon_{x}=\frac{\partial u}{\partial x}+\frac{1}{2}\left(\frac{\partial w}{\partial x}\right)^{2}, \quad \epsilon_{y}=\frac{\partial v}{\partial y}+\frac{1}{2}\left(\frac{\partial w}{\partial y}\right)^{2}, \\
\gamma_{x y}=\frac{\partial u}{\partial y}+\frac{\partial v}{\partial x}+\frac{\partial w}{\partial x} \frac{\partial w}{\partial y} .
\end{gathered}
$$

The stresses and strains are assumed, as usual, to obey the stress-strain relations

$$
\epsilon_{x}=\frac{1}{E}\left(\sigma_{x}-\nu \sigma_{y}\right), \quad \epsilon_{y}=\frac{1}{E}\left(\sigma_{y}-\nu \sigma_{x}\right), \quad \gamma_{x y}=\frac{2(1+\nu)}{E} \tau_{x y},
$$

where $E$ and $\nu$ are the modulus of elasticity and the Poisson ratio, respectively. Finally, we have the "compatibility" equation

$$
\nabla^{2}\left(\sigma_{x}+\sigma_{y}\right)=E\left\{\left(\frac{\partial^{2} w}{\partial x \partial y}\right)^{2}-\frac{\partial^{2} w}{\partial x^{2}} \frac{\partial^{2} w}{\partial y^{2}}\right\}
$$

in which $\nabla^{2}$ is the Laplace operator. ${ }^{7}$ This equation is an integrability condition for Eqs. (1.3), expressed in terms of $\sigma_{x}, \sigma_{y}$, and $w$ by the use of (1.1) and the stress-strain relations.

Two different kinds of conditions will be considered at the boundary $C$ of the sheet. In one case we prescribe the displacements $\bar{u}, \bar{v}, \vec{w}$ at the boundary;

$$
\bar{u}=u(C), \quad \bar{v}=v(C), \quad \bar{w}=0 .
$$

In the other case, instead of the displacements $\bar{u}$ and $\bar{v}$ we prescribe the normal and shear stresses $\bar{\sigma}_{n}$ and $\bar{\tau}_{n}$ at the boundary;

$$
\bar{\sigma}_{n}=\sigma_{n}(C), \quad \bar{\tau}_{n}=\tau_{n}(C), \quad \bar{w}=0 .
$$

That we may impose the boundary conditions (1.6) is clear; the differential equations (1.1), (1.2), and (1.4) together with the boundary conditions (1.6) constitute the complete formulation of a boundary value problem for the determination of the functions $\sigma_{x}, \sigma_{y}, \tau_{x y}$ and $w$. That the conditions (1.5) may be imposed could be seen readily by formulating our problem in terms of the displacements $u, v, w$ alone, but we refrain from doing so here. The conditions (1.5) mean that the edge of the sheet is stretched in its plane by a fixed amount, which does not depend upon the applied normal pressure $p$. The conditions (1.6), on the other hand, mean that the stress at the edge is held fixed while the displacements there will depend upon $p$.

- Notice that of the three quadratic terms occuring in the usual expression for the strains, only the one involving $w$ is retained in (1.3). The motivation for this is that the order of magnitude of the displacement $w$ normal to the sheet can be expected to differ from that of the displacement parallel to the plane of the sheet. The experimental results (see the paper by Eck [4]) confirm the validity of this assumption from the physical point of view.

7 These differential equations were first obtained by Föppl [6] in 1907. They can also be obtained by neglecting the terms referring to bending in the non-linear theory of plates developed by v. Kármán [11]. The equations have been solved by Hencky for the case of a circular sheet [9] and a rectangular sheet [10]. Bourgin [2] has treated the case of the rectangular sheet by methods different from those of Hencky. 
For the purpose of comparison with our new theory of curved sheets, which we develop only for the rotationally symmetric case, it is convenient for us to formulate the plane sheet theory in polar coordinates assuming all quantities to depend only on the coordinate $r$.

The differential equations corresponding to (1.1), (1.2), and (1.4) are for this case,

$$
\begin{gathered}
\frac{d}{d r}\left(r \sigma_{r}\right)=\sigma_{\phi}, \\
\frac{1}{r} \frac{d}{d r}\left(r \sigma_{r} \frac{d w}{d r}\right)=-p / h, \\
-\frac{d}{d r}\left(r \sigma_{\phi}\right)+\sigma_{r}=\frac{E}{2}\left(\frac{d w}{d r}\right)^{2} .
\end{gathered}
$$

For the purpose we have in view it is not necessary to write down the strain-displacement and stress-strain relations in polar coordinates. The boundary conditions (1.5) at the edge $r=R$ become

$$
\bar{u}=u(R), \quad \bar{w}=0,
$$

in which $\bar{u}$ refers to the radial displacement at the edge. The alternate boundary conditions (1.6) become

$$
\bar{\sigma}_{r}=\sigma_{r}(R), \quad \bar{w}=0 .
$$

We consider three different specializations of the non-linear plane sheet theory as a basis for comparison with the theory of curved sheets to be developed later. These are: Case (a), a direct linearization of the differential equations; Case (b), the classical linear membrane theory; Case (c), the problem of Föppl-Hencky. We proceed to discuss these three cases in order.

CASE (a). A direct linearization of the differential equations. If we simply neglect the non-linear terms in (1.2) and (1.3) we obtain the relations $\nabla^{2}\left(\sigma_{x}+\sigma_{y}\right)=0, p=0$. The sheet is therefore not deflected laterally; it is simply in a state of plane stress. From our point of view, such a linearization thus leads to a "trivial" problem.

It is worth while to point out that the solutions for Case (a) are also solutions of the non-linear sheet theory if we impose the condition that the normal pressure $p$ be everywhere zero.

CASE (b). The classical linear membrane theory. The well-known linear theory of tightly stretched plane membranes can be obtained from the non-linear sheet theory as an approximation to the solution of the boundary value problem in a special case. The approximation, as we shall see, results from a development in the neighborhood of Case (a). The special case of the non-linear theory in question arises when the boundary condition is taken in the form (1.6) with $\bar{\sigma}_{n}$ assumed to be a constant $\bar{\sigma}>0$, $\bar{\tau}_{n}$ to be zero:

$$
\bar{\sigma}_{n}=\bar{\sigma}>0, \quad \bar{\tau}_{n}=0, \quad \bar{w}=0 .
$$

Furthermore, we make the important additional assumption that the applied normal pressure $p$ is small compared with $\bar{\sigma}$. In other words we assume the membrane to be tightly stretched and then deflected by a relatively small normal pressure.

We can solve this boundary value problem by a perturbation method consisting of a development in the neighborhood of the solution for the case in which $w=0$, 
$p=0$ (that is, in the neighborhood of the undeflected state of the stretched sheet). The well-known linear membrane theory results as the second step in such a development. We need only develop $\sigma_{x}, \sigma_{y}, \tau_{x y}$, and $w$ in terms of the parameter $\epsilon$ defined by $\epsilon=p / \bar{\sigma}$, as follows:

$$
\left.\begin{array}{l}
\sigma_{x}=\sigma_{x}^{(0)}+\epsilon^{2} \sigma_{x}^{(2)}+\cdots, \quad \sigma_{y}=\sigma_{\nu}^{(0)}+\epsilon^{2} \sigma_{\nu}^{(2)}+\cdots, \\
\tau_{x y}=\tau_{x y}^{(0)}+\epsilon^{2} \tau_{x y}^{(2)}+\cdots, \quad w=\epsilon w^{(1)}+\epsilon^{3} w^{(2)}+\cdots, \quad p=\epsilon \bar{\sigma} .
\end{array}\right\}
$$

The stresses (including the stress $\bar{\sigma}$ at the boundary) are of lower order in $\epsilon$ than the deflection $w$ and the applied pressure $p$. Insertion of relations (1.8) in the differential equations (1.1), (1.2), (1.4) and the boundary conditions (1.7) leads to a sequence of linear boundary value problems for the determination of the coefficients in the perturbation series. For the terms of zero order in the stresses one finds readily the solution $\sigma_{x}^{(0)}=\sigma_{y}^{(0)} \equiv \bar{\sigma}, \tau_{x y}^{(0)} \equiv 0$; in other words the zero order terms represent a state of uniform tension throughout the sheet. The zero order terms are also, evidently, the solution for the linearized sheet theory of Case (a). The differential equation for $w^{(1)}$ is then readily found to be

$$
\nabla^{2} w^{(1)}=-1 / h,
$$

while the boundary condition is, of course,

$$
w^{(1)}(C)=0 .
$$

Equations (1.9) and (1.10) are those of the classical linear membrane theory (for unit normal pressure). For the applicability of this theory the essential condition is that the applied pressure $p$ should be small compared with the initial stress $\bar{\sigma}$ in the sheet. We note also that this theory results when the stress is prescribed at the boundary rather than the displacement in the plane of the sheet; in other words, the linear membrane theory requires that the edge of the sheet be free to move in the $x y$-plane.

CaSE (c). The problem of Föppl-Hencky. The boundary value problem which leads to our Case (c) is that resulting from the choice of (1.5) as boundary conditions for the non-linear sheet theory. This theory is sometimes referred to as the large deflection theory of membranes. It is not assumed, as in the above Case (b), that the normal pressure $p$ is small compared with the initial stress in the sheet. In fact, we assume for the Case (c) that the displacements $\bar{u}$ and $\bar{v}$ at the boundary as well as $\bar{w}$ are zero. We shall refer to this problem ${ }^{8}$ on occasion as the problem of Föppl-Hencky. Our boundary conditions of course mean that the sheet was initially unstrained. Thus the stresses in the sheet are built up only as the normal pressure $p$ is applied, and consequently the procedure outlined above for Case (b) is entirely inapplicable.

As already stated, our purpose is to generalize the non-linear sheet theory $(c)$ to the case of curved sheets. The essential step for this purpose consists in developing suitable non-linear strain relations for the curved sheet analogous to those (cf. (1.3)) for the plane sheet. However it is not entirely clear a priori in the case of curved sheets just which of the quadratic terms in the strain equations should be retained and which rejected. Section 2 is devoted to a derivation and discussion of the strain expressions

8 The previously cited papers of Hencky $[9,10]$ and Bourgin [2] are concerned with this problem. The problems for the case in which the boundary displacements $\bar{u}$ and $\bar{v}$ are not zero (i.e. the case of an initially stretched sheet) appear not to have been treated. 
used later as the basis for our theory. The discussion is confined to the case of the rotationally symmetric deformation of a surface of revolution. Only two displacements are involved in this case, the displacement $u$ along a meridian and the displacement $w$ along the normal to the sheet.

Once expressions for the strains in terms of the displacements are available, it becomes possible to set up the integral for the potential energy in the sheet (assuming Hooke's law to hold) in terms of the displacements $u$ and $w$. The equilibrium conditions can then be found as the Euler variational equations minimizing the potential energy. The result is a pair of second order non-linear differential equations for $u$ and $w$ which permit the boundary condition $\bar{u}=\bar{w}=0$ for a fixed edge to be imposed.

For most purposes it is, however, more convenient to formulate the curved sheet theory in terms of the two components $\sigma_{\theta}$ and $\sigma_{\phi}$ of the stress in the sheet along and perpendicular to a meridian curve, respectively, and the displacement $w$ normal to the sheet, rather than in terms of the two displacements $u$ and $w$. This is carried out in Section 3. In Section 4 the general theory is specialized for the case of the spherical sheet. The result is a set of differential equations for the curved sheet analogous to (1.1) and (1.2) for the plane sheet. In Section 5 a simplification in the theory for the spherical sheet is introduced which is valid for a spherical segment of small curvature (and probably also for all cases of spherical sheets). The differential equations of Section 5 are

$$
\begin{gathered}
\frac{d}{d \theta}\left(\sigma_{\theta} \sin \theta\right)=\sigma_{\phi} \cos \theta, \\
\frac{1}{R} \frac{d}{d \theta}\left(\sigma_{\theta} \sin \theta \frac{d w}{d \theta}\right)=-\left(\frac{R p}{h}+\sigma_{\phi}+\sigma_{\theta}\right) \sin \theta, \\
-\frac{d}{d \theta}\left(\sigma_{\phi} \tan \theta\right)+\left(1+\nu \tan ^{2} \theta\right) \sigma_{\theta} \\
=\frac{E}{R}\left\{w \tan ^{2} \theta+\tan \theta \frac{d w}{d \theta}+\frac{1}{2 R}\left(\frac{d w}{d \theta}\right)^{2}\right\} .
\end{gathered}
$$

The independent variable $\theta$ is the latitude angle measured from the pole of the sphere. These equations are exactly analogous to Eqs. $(1.1)^{\prime},(1.2)^{\prime}$, and $(1.4)^{\prime} .{ }^{9}$ We refrain from writing the stress-strain and strain-displacement relations which are needed for a complete formulation of the problem.

As boundary conditions at $\theta=\theta_{0}$ we assume either

or

$$
\bar{u}=u\left(\theta_{0}\right), \quad \bar{w}=w\left(\theta_{0}\right),
$$

$$
\bar{\sigma}_{\theta}=\sigma_{\theta}\left(\theta_{0}\right), \quad \bar{w}=w\left(\theta_{0}\right) .
$$

At the pole $\theta=0$ we require all quantities to remain finite.

We wish to consider the three special cases in connection with Eqs. (1.11) which are analogous to the three cases discussed above in connection with the plane sheet. These are: Case (a), a direct linearization; Case (b), the analogue for curved sheets of

- If we were to allow $R$ to tend to infinity while $\theta$ tends to zero in (1.11) in such a way that $R \theta \rightarrow r$, the result would be the differential equations $(1.1)^{\prime},(1.2)^{\prime}$ and $(1.4)^{\prime}$. (The normal pressure $p$ and the displacement $w$ are taken positive in the direction toward the center of the sphere.) 
the classical linear membrane theory; Case $(c)$, the analogue of the Föppl-Hencky problem for curved sheets. We consider these cases in order.

CASE (a). A direct linearization. In contrast to the corresponding case in the plane sheet theory, we observe that neglect of the non-linear terms in (1.11) does not lead to a trivial problem. We obtain, in fact, the equations

$$
\left.\begin{array}{c}
\frac{d}{d \theta}\left(\sigma_{\theta} \sin \theta\right)=\sigma_{\phi} \cos \theta, \quad \sigma_{\theta}+\sigma_{\phi}=-\frac{R p}{h}, \\
\left(\sigma_{\phi} \tan \theta\right)+\left(1+\nu \tan ^{2} \theta\right) \sigma_{\theta}=\frac{E}{R}\left(w \tan ^{2} \theta+\tan \theta \frac{d w}{d \theta}\right) .
\end{array}\right\}
$$

These are the differential equations of what is called the "membrane theory" of thin shells. One observes that the order of the system (1.11)' is two less than that of (1.11) The stresses can be obtained from the first two equations of $(1.11)^{\prime}$ without reference to the strains and displacements. It is, however, not possible to satisfy in general the kinds of boundary conditions which would be natural in the physical situations encountered in the applications. One such case is that of a fixed edge, which would imply the condition $w=0$ at the boundary. That this condition cannot be satisfied, at least in the case $p=$ const., is readily seen : the only solution of $(1.11)^{\prime}$ that is finite at $\theta=0$ is given by

$$
\sigma_{\theta}=\sigma_{\phi}=-\frac{p R}{2 h}, \quad w=p R^{2}(1-\nu) / 2 E h .
$$

All three quantities are constant throughout the shell.

As in the corresponding Case (a) for the plane sheet, the solution (1.14) of the linear equations $(1.11)^{\prime}$ is also a solution of the non-linear equations (1.11) if proper restrictions are imposed. Instead of prescribing the boundary values $\bar{\sigma}_{\theta}$ and $\bar{w}$ in (1.13) arbitrarily, we would require them to have values consistent with (1.14).

CASE (b). The analogue for curved sheets of the classical linear membrane theory. The theory of curved sheets analogous to the classical linear membrane theory for plane sheets seems not to have been developed. For the case of a spherical sheet we can obtain such a theory from Eqs. (1.11) with the boundary condition (1.13), in which, however, we assume $\bar{\sigma}_{\theta}$ and $\bar{w}$ to have values consistent with (1.14) and set $p=p^{(0)}$. However, we assume that the pressure $p$ in (1.11) is given by

$$
p=p^{(0)}+\epsilon p^{(0)}
$$

in which $\epsilon$ is a small (and, of course, dimensionless) parameter. The theory we desire then results from the terms of first order in the development of the solution by perturbations with respect to $\epsilon$. We set

$$
\left.\begin{array}{rl}
w & =w^{(0)}+\epsilon w^{(1)}+\epsilon^{2} w^{(2)}+\cdots, \\
\sigma_{\theta} & =\sigma_{\theta}^{(0)}+\epsilon \sigma_{\theta}^{(1)}+\cdots, \\
\sigma_{\phi} & =\sigma_{\phi}^{(0)}+\epsilon \sigma_{\phi}^{(1)}+\cdots,
\end{array}\right\}
$$

and insert these series together with (1.15) in the differential equations (1.11) and the boundary conditions

$$
\bar{\sigma}_{\theta}=\bar{\sigma}_{\phi}=-p^{(0)} R / 2 h, \quad \bar{w}=p^{(0)} R^{2}(1-\nu) / 2 E h .
$$


The terms of zero order in (1.16) are then readily found to be those which would result from the linear theory-in other words $\sigma_{\theta}^{(0)}, \sigma_{\phi}^{(0)}$, and $w^{(0)}$ have throughout the sheet the constant values prescribed at the boundary in (1.17). The first order terms are then found to satisfy the linear differential equations

$$
\left.\begin{array}{rl}
\frac{d}{d \theta}\left(\sigma_{\theta}^{(1)} \sin \theta\right) & =\sigma_{\phi}^{(1)} \cos \theta \\
\frac{1}{R} \frac{d}{d \theta}\left(\sigma_{\theta}^{(0)} \sin \theta \frac{d w^{(1)}}{d \theta}\right) & =-\left(\frac{R p^{(0)}}{h}+\sigma_{\theta}^{(1)}+\sigma_{\phi}^{(1)}\right) \sin \theta, \\
-\frac{d}{d \theta}\left(\sigma_{\phi}^{(1)} \tan \theta\right)+\left(1+\nu \tan ^{2} \theta\right) \sigma_{\theta}^{(1)} & =\frac{E}{R}\left(\tan \theta \frac{d w^{(1)}}{d \theta}+w^{(1)} \tan ^{2} \theta\right),
\end{array}\right\}
$$

and the boundary conditions

$$
\bar{\sigma}_{\theta}^{(1)}=0, \quad \bar{w}^{(1)}=0 .
$$

Equations (1.18) and (1.19) are analogous to (1.9) and (1.10) for the corresponding case of the plane sheet. It must, however, be admitted that this "theory of tightly stretched membranes" for the sphere is somewhat artificial because of the fact that the "stretched" state is one for which the initial radial displacement $w$ cannot be held zero at the boundary.

CASE (c). The analogue of the Föppl-Hencky problem for curved sheets. The differential equations (1.11) are to be solved for a prescribed pressure $p$ when the edge of the sheet is considered fixed, i.e., under the boundary conditions $\bar{w}=0$ and $\bar{u}=0$. In this particular case the condition $\bar{u}=0$ can be replaced by the condition that the strain in the direction of the boundary curve is zero, which implies the condition $\bar{\sigma}_{\phi}-\nu \bar{\sigma}_{\theta}=0$ on $\sigma_{\phi}$ and $\sigma_{\theta}$ at the boundary. The analogy with the corresponding case for the plane sheet is, as we see, exact in every respect.

It should now be apparent that some such term as "sheet theory" is needed in addition to the term "membrane theory." This is brought out by Table I which lists the Cases (a), (b), and (c) together with the present terminology. As we note, the phrase "membrane theory" is already applied to cases which have almost nothing in common. Consequently we would recommend (in accordance with a suggestion made by Bourgin [2]) that all of these theories which neglect bending be referred to in

TABLE I.

\begin{tabular}{l|l|c}
\hline Cases & \multicolumn{1}{|c|}{ Plane } & \multicolumn{1}{c}{ Curved } \\
\hline (a) & Plane stress & Membrane theory of shells \\
\hline (b) & Membrane with small deflections & - \\
\hline (c) & Large deflection theory of membranes & - \\
\hline
\end{tabular}

general as sheet theories. The Cases (a) and (c) could then be referred to as linear and non-linear sheet theories respectively, while the term membrane theory might be reserved for the Cases (b), i.e., for theories of initially stretched sheets which start with 
the linear sheet theory as a first approximation and then proceed to a sccond approximation by a development in the neighborhood of the solution to the linearized problem. This terminology will be used in the remainder of this paper.

In Section 3, the differential equations for the curved sheet theory are obtained, as we have already indicated, by variational methods. In that section also, the stability of the extremal solutions for both the linear and the non-linear sheet theories [Cases (a) and (c)] is considered. At first sight one would be inclined to think that the solutions in the two cases would not differ greatly as far as stability is concerned if the pressure, thickness, etc., are the same in both cases. This is, however, not true. On the basis of the linear curved sheet theory, the solutions would appear to be stable whether the pressure $p$ is inward or outward, that is, whether the sheet is in compression or tension, respectively. On the basis of the non-linear curved sheet theory, however, the solutions are unstable when the pressure is such as to cause the stress $\sigma_{\theta}$ in the sheet to be a compression. ${ }^{10}$ This result follows through consideration of the Legendre condition for our variational problem. In the case of the spherical sheet the $\operatorname{stress} \sigma_{\theta}$, as given by the linear theory, is a compression when the normal pressure $p$ is positive (i.e., when the pressure is directed toward the center of the sphere). It also seems certain that the non-linear theory will yield the same relation between the signs of $\sigma_{\theta}$ and $p$ for the case of the spherical sheet, unless the displacements are very large. Consequently, we have assumed in our numerical work that the pressure $p$ is negative, i.e., is directed outward, in order to avoid unstable cases.

In Section 5 the non-linear curved sheet theory [Case (c)] is formulated in detail for the special case of a spherical segment of small curvature. The differential equations for the spherical sheet can be solved by power series in the independent variable. Graphs showing the distribution of the stresses and the normal deflection $w$ along a meridian in a particular numerical case are given in Section 6. Perhaps the most striking feature of these results is that the non-linear sheet theory [Case (c)] yields results which do not differ greatly from those of the linear theory [Case (a)] except near the edge of the shect. In particular, the stresses and the normal displacement $w$ are nearly constant over most of the interior of the sheet, but change rather rapidly near its edge.

This observation indicates that we have to deal here with a boundary layer effect. In Section (7) the existence of such an effect is deduced and treated explicitly. It turns out upon introduction of proper dimensionless variables in the original differential equations that only one parameter $\kappa$ remains in the transformed differential equations. The quantity $\kappa$ is given by

$$
\kappa=p R / E h,
$$

in which $p$ is the normal pressure on the sheet, $R$ the radius, $E$ the modulus of elasticity, and $h$ the thickness of the sheet. If $\kappa$ is allowed to tend to zero in the transformed differential equations the result is in the limit the differential equations of the linear sheet theory with a consequent lowering of the order of the system. Hence some boundary condition must be lost at the edge on the transition to the value $\kappa=0$. The solutions of the boundary value problem for $k \neq 0$ can therefore not be expected to converge uniformly at the boundary to the solution of the problem for $\kappa=0$. It is

${ }^{10}$ Comparison with the analogous cases (a) and (c) for the plane sheet theory is illuminating. It is clear that the plane sheet would be stable under edge compression if no lateral deflection were to be permitted, but decidedly unstable under compression if such a lateral constraint were not imposed. 
possible to treat the boundary layer phenomenon by introducing a new independent variable which depends upon $\kappa$ in such a way as to stretch the boundary layer to infinity as $\kappa \rightarrow 0$, with the result that the convergence of the solutions becomes uniform with respect to $\kappa$ at the edge. One notices that the value $\kappa=0$ corresponds, according to $(1.20)$, to the value zero for the pressure $p$.

The boundary layer solution is given in this case very simply by an exponential function. It could be used to estimate the stresses in practice in cases for which $\kappa$ is small (and, of course, negative). In the usual cases it is not difficult to see that $\kappa$ will be of the order of -0.0005 in practice, since $p R / 2 h$ is the stress when $p$ is constant, according to the linear theory, and hence $\kappa$ is a quantity of the order of the longitudinal strains. ${ }^{11}$

It is clear that the non-linear sheet theory could be worked out in detail rather readily in other cases such as those of the cylindrical and conical sheets. It would also be of interest to consider the case of the spherical sheet with a hole, so that two distinct boundary curves $y$ would exist. Various combinations of boundary conditions at the two edges should be considered; boundary layer effects could then occur at both edges.

From the point of view of the practical applications, another question is of interest. It is clear that bending effects will dominate the "sheet effects" near the edge of the sheet if the sheet is thick enough. This question is under investigation at the present time.

2. Expressions for the longitudinal strains. We assume the curved sheet to be the surface of revolution obtained by rotating about the $y$-axis the meridian curve $C$,

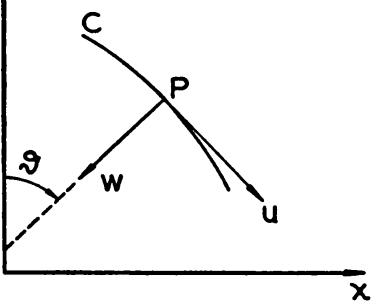

Fig. 2.

$$
x=x(\xi), \quad y=y(\xi) .
$$

The parameter $\xi$ is taken to be the arc length of the curve. We consider only deformations which preserve rotational symmetry, so that the deformation is completely described by the displacement components $u$ and $w$ along the meridian and along the normal to the surface respectively.

It is convenient to introduce the angle $\theta$ between the $y$-axis and the normal to the meridian. These notations are indicated in Fig. 2.

The longitudinal strains in the sheet are defined in the usual way. If $d s^{1}$ is the deormed length of the line element originally of length $d s$, then the strain $\epsilon$ in the direction of the element $d s$ is defined by

$$
\left(\frac{d s^{1}}{d s}\right)^{2}=1+2 \epsilon
$$

[ $t$ is useful to introduce the following relations between the original position $(x, y, z)$ and the deformed position $\left(x^{1}, y^{1}, z^{1}\right)$ of any point $P$ on the sheet:

11 The effect of the edge constraint seems to be such as to cause the stresses at the edge to be lower in ralue than in the interior of the sheet. Thus it seems likely that the usual practice in engineering design if ignoring the edge effect leads to estimates for the stresses which are too high, i.e., are on the side of afety. Of course, we are entitled to draw this conclusion here only in case the sheet is in tension. 


$$
x^{1}=x+u \cos \theta-w \sin \theta, \quad y^{1}=y-u \sin \theta+w \cos \theta, \quad z^{1}=z .
$$

By making use of these relations the strains $\epsilon_{\theta}$ and $\epsilon_{\phi}$ in the direction of a meridian and a parallel (i.e., a curve $\theta=$ const.) are easily computed by using (2.2). We obtain

$$
\begin{aligned}
& \epsilon_{\theta}=\frac{1}{\rho}\left(\frac{d u}{d \theta}-w\right)+\frac{1}{2 \rho^{2}}\left(u+\frac{d w}{d \theta}\right)^{2}+\frac{1}{2} \frac{1}{\rho^{2}}\left(\frac{d u}{d \theta}-w\right)^{2}, \\
& \epsilon_{\phi}=\frac{u \cos \theta-w \sin \theta}{x}+\frac{1}{2}\left(\frac{u \cos \theta-w \sin \theta}{x}\right)^{2} .
\end{aligned}
$$

The quantity $\rho$ is the radius of curvature of the meridian curve and $x$ is, of course, the abscissa of the point $P$.

Just as is done in the analogous case of the plane sheet, we retain only certain of the quadratic terms in the strain expressions, which then amounts to the assumption that these non-linear terms are considered to be of the same order as the linear terms. Thus it would be logical to reject the third term on the right hand side of (2.3) and the second term on the right hand side of (2.4), since they are squares of the linear terms. We shall follow this procedure and thus take for the strains the expressions

$$
\begin{aligned}
\epsilon_{\theta} & =\frac{1}{\rho}\left(\frac{d u}{d \theta}-w\right)+\frac{1}{2 \rho^{2}}\left(u+\frac{d w}{d \theta}\right)^{2}, \\
\epsilon_{\phi} & =\frac{u \cos \theta-w \sin \theta}{x} .
\end{aligned}
$$

The following special cases are of interest:

a) The sphere. ${ }^{12}$ Here $\rho=R$ (the radius of the sphere), and we find from (2.5) and (2.6) that

$$
\begin{aligned}
\epsilon_{\theta} & =\frac{1}{R}\left(\frac{d u}{d \theta}-w\right)+\frac{1}{2 R^{2}}\left(\frac{d w}{d \theta}+u\right)^{2}, \\
\epsilon_{\phi} & =\frac{1}{R}(u \cot \theta-w) .
\end{aligned}
$$

b) The circular cylinder. Here $\rho=\infty, \theta=\pi / 2, x=a$ (the radius of the cylinder), and $\xi=y$. We find that

$$
\epsilon_{y}=\frac{d u}{d y}+\frac{1}{2}\left(\frac{d w}{d y}\right)^{2}, \quad(2.9) \quad \epsilon_{\phi}=-\frac{w}{a} .
$$

c) The circular cone. Here $\rho=\infty, \theta=\gamma=$ const. The strains are given by

$$
\begin{aligned}
\epsilon_{\theta} & =\cos \gamma \frac{d u}{d x}+\frac{1}{2} \cos ^{2} \gamma\left(\frac{d w}{d x}\right)^{2}, \\
\epsilon_{\phi} & =\frac{u \cos \gamma-w \sin \gamma}{x} .
\end{aligned}
$$

12 These expressions coincide with those used by Friedrichs [7]. Similar expressions were used earlier by Biezeno [1]. 
3. Formulation of the boundary value problem for a sheet with fixed edges. On the assumption that Hooke's law holds for the relation between the strains $\epsilon_{\theta}$ and $\epsilon_{\phi}$ defined above and the corresponding normal stresses $\sigma_{\theta}$ and $\sigma_{\phi}{ }^{13}$ we have for the potential energy $V$ of the sheet the expression

$$
V=2 \pi \int_{\xi_{1}}^{\xi_{0}}\left(\frac{1}{2} \frac{E h}{1-\nu^{2}}\left[\epsilon_{\theta}^{2}+\epsilon_{\phi}^{2}+2 \nu \epsilon_{\theta} \epsilon_{\phi}\right]-p w\right) x d \xi,
$$

in which $p$ is the normal pressure on the sheet counted positive in the direction of the inward normal. The quantities $\xi$ and $x(\xi)$ are the arc length and abscissa of the meridian curve, and $h$ is the thickness of the sheet. The quantities $\nu$ and $E$ are the elastic constants.

The potential energy could be expressed in terms of the displacements $u$ and $w$ by replacing $\epsilon_{\theta}$ and $\epsilon_{\phi}$ in terms of these quantities through (2.5) and (2.6). The variational equations for the minimum problem would then clearly be a pair of non-linear ordinary differential equations for $u$ and $w$, each of which would be of the second order. We shall not write these equations down since in the following we wish to work with the stresses $\sigma_{\theta}$ and $\sigma_{\phi}$, and the displacement $w$ as dependent variables. However, we do wish to draw one conclusion from the existence of two such equations. The differential equations for $u$ and $w$ are of the proper order to permit imposition of the boundary condition $u=w=0$ appropriate for a fixed edge.

The variational equations resulting from (3.1) are

$$
\begin{array}{r}
\frac{1}{x} \frac{d}{d \xi}\left(x \sigma_{\theta}\right)-\left[\frac{\sigma_{\theta}}{\rho}\left(\frac{d w}{d \xi}+\frac{u}{\rho}\right)+\frac{\sigma_{\phi} \cos \theta}{x}\right]=0, \\
\frac{1}{x} \frac{d}{d \xi}\left[x \sigma_{0}\left(\frac{d w}{d \xi}+\frac{u}{\rho}\right)\right]+\left[\frac{\sigma_{\theta}}{\rho}+\frac{\sigma_{\phi} \sin \theta}{x}+\frac{p}{h}\right]=0 .
\end{array}
$$

The quantity $\rho$ in these equations represents the radius of curvature of the meridian curve; the quantities $\rho, \theta$, and $x$ are, of course, given functions of $\xi$. In deriving (3.2) and $(33)$ use was made of the stress-strain relations

$$
E \epsilon_{\theta}=\sigma_{\theta}-\nu \sigma_{\phi}, \quad E \epsilon_{\phi}=\sigma_{\phi}-\nu \sigma_{\theta},
$$

and of (2.5) and (2.6) in order to introduce $\sigma_{\theta}$ and $\sigma_{\phi}$ as dependent variables. Equations (3.2), (3.3), (3.4), (2.5), and (2.6), together with appropriate boundary conditions, yield the complete formulation of the boundary value problems we consider here. We note that there are six equations for the six quantities $u, w, \sigma_{\theta}, \sigma_{\phi}, \epsilon_{\theta}, \epsilon_{\phi}$.

For the most part, we are concerned with the case of a sheet without a hole at the axis of symmetry, so that the quantity $x$ in (3.2) and (3.3) has the value zero where the meridian curve crosses the axis, which we may assume to occur for $\xi=0$. In this case we would require the solution to be regular at $\xi=0$. At an edge $\xi=\xi_{0}$ of the sheet we require $u=w=0$, for a fixed edge. In view of (2.6) we see that this implies $\epsilon_{\phi}=0$; hence we may prescribe the following conditions at a fixed edge:

$$
\text { at } \xi=\xi_{0}\left\{\begin{aligned}
w & =0 \\
E \epsilon_{\phi} & =\sigma_{\phi}-\nu \sigma_{\theta}=0 .
\end{aligned}\right.
$$

In this way we express the boundary condition in terms of $w, \sigma_{\theta}$, and $\sigma_{\phi}$.

${ }^{13}$ On account of symmetry the shear stresses in the coordinate directions are of course zero. 
We remark that the so-called membrane theory of axially symmetric shells results from the above theory when all non-linear terms in $\sigma_{\theta}, \sigma_{\phi}, u$, and $w$ are rejected.

We have already stated in the introduction that the solutions of the variational equations (3.2) and (3.3) are unstable when the "radial" stress $\sigma_{\theta}$ is negative (i.e., when it is a compressive stress). On the other hand, it was stated that the solutions of the linear sheet theory are stable whether $\sigma_{\theta}$ is positive or negative. The conclusion regarding the instability in the non-linear case results immediately from the fact that the Legendre condition on the second variation of $V$ is not satisfied if $\sigma_{\theta}$ is negative, which means that the extremals do not render $V$ a minimum in this case. The Legendre condition ${ }^{14}$ for a minimum in our case requires that the quantity $\Delta$ given by

$$
\Delta=F_{u_{\xi} u_{\xi}} F_{w_{\xi} w_{\xi}}-F_{u_{\xi} w_{\xi}}^{2}
$$

should be positive. The quantity $F$ is the integrand in (3.1) and subscripts denote differentiations. It turns out that the quantity $\Delta$ can be expressed in the form $\Delta=4 \pi^{2} h^{2} E\left(1-\nu^{2}\right)^{-1} x^{2} \sigma_{\theta}$. The right hand side has the sign of $\sigma_{\theta}$, and consequently the Legendre condition is violated at all points where $\sigma_{\theta}$ is negative.

In the special case of the spherical sheet, it is possible to put the sign of $\sigma_{\theta}$ in relation to that of the applied pressure $p$. If the boundary conditions are specialized in such a way that the solution of the linear sheet theory results, we know [cf. (1.14)] that $p$ and $\sigma_{\theta}$ are opposite in sign, so that the solutions in this case are unstable when $p$ is positive, i.e., when $p$ is directed toward the center of the sphere. Since it is not possible to give the solutions explicitly in the general non-linear case, we have not been able to prove readily that $\sigma_{\theta}$ and $p$ are opposite in sign in this case; but if the displacements remain small there can be little doubt that $p$ and $\sigma_{\theta}$ differ in sign in these cases also. In our further discussion of the spherical sheet we have therefore assumed always that $p$ is negative, i.e., that it is directed outward from the center of the sphere.

The linearized sheet theory results from (3.1) when all terms of degree higher than the second in $u$ and $w$ and their derivatives are neglected at the outset. If this is done, the Legendre condition for the resulting variational problem becomes $\Delta=F_{u \xi u \xi}>0$, with

$$
\Delta=\frac{2 \pi x E h}{1-\nu^{2}}
$$

which is always positive, since $x$ (the coordinate measuring the distance from the axis of the sheet) is always positive. Hence the Legendre condition is always satisfied in the case of the linear sheet theory, and we expect all solutions to be stable. The reason for the stable character of all solutions given by the linear theory, as contrasted with the unstable character of some of the solutions given by the non-linear theory, is that the linearization is equivalent to the imposition of a constraint powerful enough to cause stability in all cases.

4. The spherical sheet. In the special case of the sphere we may write $\xi=R \theta$, $p=R$, and $x=R \sin \theta, R$ being the radius of the sphere. The differential equations for the sphere are

${ }^{14}$ See, for example, R. Courant and D. Hilbert, Methoden der mathematischen Physik, vol. 1, Julius Springer, Berlin, 1931, p. 184. 


$$
\begin{gathered}
\frac{d}{d \theta}\left(\sigma_{\theta} \sin \theta\right)=\frac{1}{R}\left(\frac{d w}{d \theta}+u\right) \sigma_{\theta} \sin \theta+\sigma_{\phi} \cos \theta, \\
\frac{1}{R} \frac{d}{d \theta}\left[\sigma_{\theta}\left(\frac{d w}{d \theta}+u\right) \sin \theta\right]=-\left(\frac{R p}{h}+\sigma_{\theta}+\sigma_{\phi}\right) \sin \theta .
\end{gathered}
$$

The system of equations is completed by the two strain-displacement relations (2.7) and (2.8) and the stress-strain relations (3.4).

As boundary conditions at a fixed edge $\theta=\theta_{0}$ we have [cf. the remarks preceding (3.5) and (3.6)]

$$
w=0,
$$

$$
\sigma_{\phi}-\nu \sigma_{\theta}=0 .
$$

In case the sheet has no hole at the axis, we require the solutions to be regular at $\theta=0$.

It is of interest to consider the special case of the complete sphere, in which the boundary conditions would become regularity conditions for $\theta=0$ and $\theta=\pi$. In case we assume that the load $p$ is constant, it is readily verified that a solution of our differential equations which satisfies the regularity conditions is $u=0, w=p R^{2}(1-\nu) / 2 E h$, $\sigma_{\theta}=\sigma_{\phi}=-p R / 2 h$. It could also be shown that this is the unique solution to this problem. We observe that this solution is identical with that furnished by the linear sheet theory (a) of shells. In other words, the non-linear terms have no influence on the solutions for the full sphere in case the applied pressure $p$ is constant. If $p$ is not constant, however, the non-linear terms will influence the results for the full sphere.

5. The spherical segment with small curvature. The differential equations of the Föppl-Hencky theory for the deflection of a radially symmetric plane sheet are contained in the above equations as a limit case. We need only allow $R$ to tend to infinity while $\theta$ approaches zero in such a way that the product $R \sin \theta$ approaches a finite limit $r$, and $r$ is thus the polar coordinate which locates points in the plane sheet. The resulting equations $(1.1)^{\prime}$ and $(1.2)^{\prime}$ have already been given in the introduction. In passing to the limit, one observes particularly that the term $u$ in the second parenthesis on the right hand side of (2.7) drops out, so that the non-linear term reduces to $\frac{1}{2}(d w / d r)^{2}$. As a consequence of this, the variational equations for the case of the plane sheet are much simpler than (4.1) and (4.2), since the terms corresponding to the first term on the right hand side of (4.1) and the term $u$ in the parenthesis on the left hand side of (4.2) disappear.

It is clear that we could also simplify our equations for the spherical sheet quite considerably by omitting the non-linear terms involving $u$ in the expression (2.7) for $\epsilon_{\theta}$. It would seem fair to expect that such a simplification would be justified for the special case of rather flat spherical sheets. We recall that the choice of the expressions for the strains in terms of the displacements was in any case somewhat arbitrary. At the beginning, we might have considered the displacement $u$ as negligible compared with the quantity $d w / d \theta$, since we expect the order of magnitude of the displacement $w$ in the direction of the applied load to differ from that of the displacement $u$. In other words, it may well be that this term could be neglected even for sheets of rather large curvature. ${ }^{15}$ In what follows we shall neglect this term.

${ }^{16}$ It might be noted that the limit problem which leads to the boundary layer phenomena (to be treated in the next section) is the same whether the terms in the displacement $u$ under discussion here are retained or not. This is another valid reason for considering these terms to be negligible in most cases. 
One result of this assumption is that the variational equations no longer contain the function $u$, but only $w, \sigma_{\theta}$, and $\sigma_{\phi}$. We can obtain a third equation in these same quantities - a "compatibility equation" - by eliminating $u$ from (2.7) and (2.8) and then replacing $\epsilon_{\theta}$ and $\epsilon_{\phi}$ by $\sigma_{\theta}$ and $\sigma_{\phi}$ through use of the stress-strain relations. The result is the system of equations

$$
\begin{aligned}
\frac{d}{d \theta}\left(\sigma_{\theta} \sin \theta\right) & =\sigma_{\phi} \cos \theta \\
\frac{1}{R} \frac{d}{d \theta}\left[\sigma_{\theta} \frac{d w}{d \theta} \sin \theta\right] & =-\left[\frac{R p}{h}+\sigma_{\theta}+\sigma_{\phi}\right] \sin \theta \\
-\frac{d}{d \theta}\left(\sigma_{\phi} \tan \theta\right)+\left(1+\nu \tan ^{2} \theta\right) \sigma_{\theta} & =\frac{E}{R}\left[w \tan ^{2} \theta+\tan \theta \frac{d w}{d \theta}+\frac{1}{2 R}\left(\frac{d w}{d \theta}\right)^{2}\right] .
\end{aligned}
$$

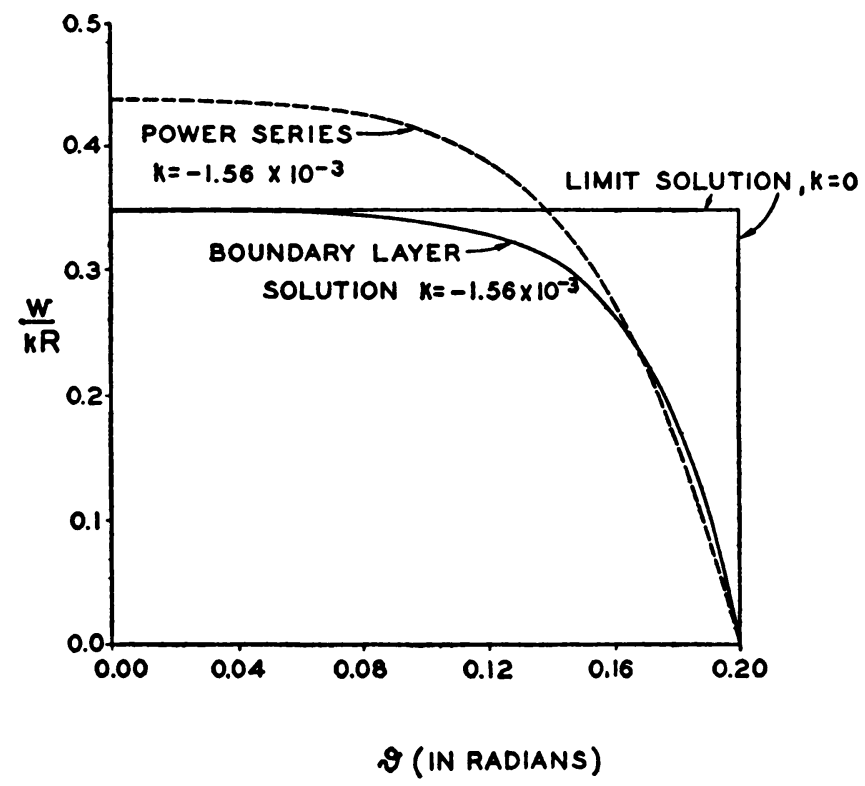

Fig. 3. Normal displacement.

These equations are identical with Eqs. (1.11) which served as the basis for the discussion of the curved sheet theory in the introduction.

We are interested in solving the differential equations (5.1), (5.2), (5.3) for the case of a spherical segment without a hole about the axis $\theta=0$ and with a fixed edge at $\theta=\theta_{0}$. This means that we require the solution to be regular at $\theta=0$ and to satisfy at the edge $\theta=\theta_{0}$ the conditions

$$
w=0 \text {, }
$$

$$
\sigma_{\phi}-\nu \sigma_{\theta}=0 .
$$

6. Numerical solution of the boundary value problem for the flat spherical segment. Our principal object in this paper is to present a new theory of thin sheets and to compare and contrast it with other theories, rather than to give numerical solutions for the resulting boundary value problems. However, we have obtained approxi- 
mate numerical solutions of the boundary value problem formulated in Eqs. (5.1) to (5.5) of the preceding section, and will report the results briefly in this section.

The graphs of Figs. 3, 4, and 5 indicate the results of an approximate solution ${ }^{16}$

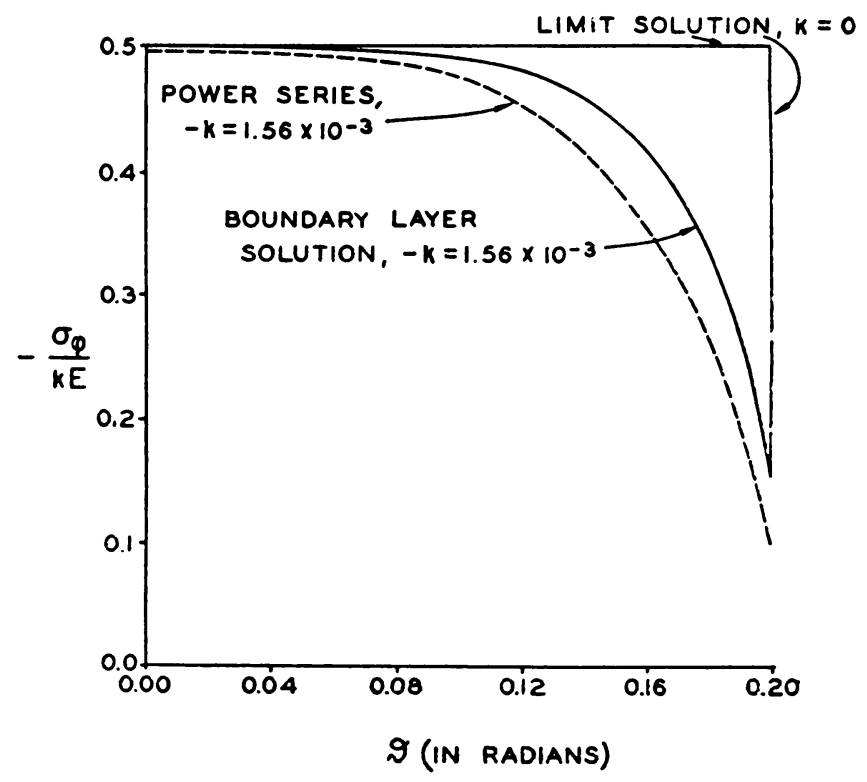

FIG. 4. Circumferential stress.

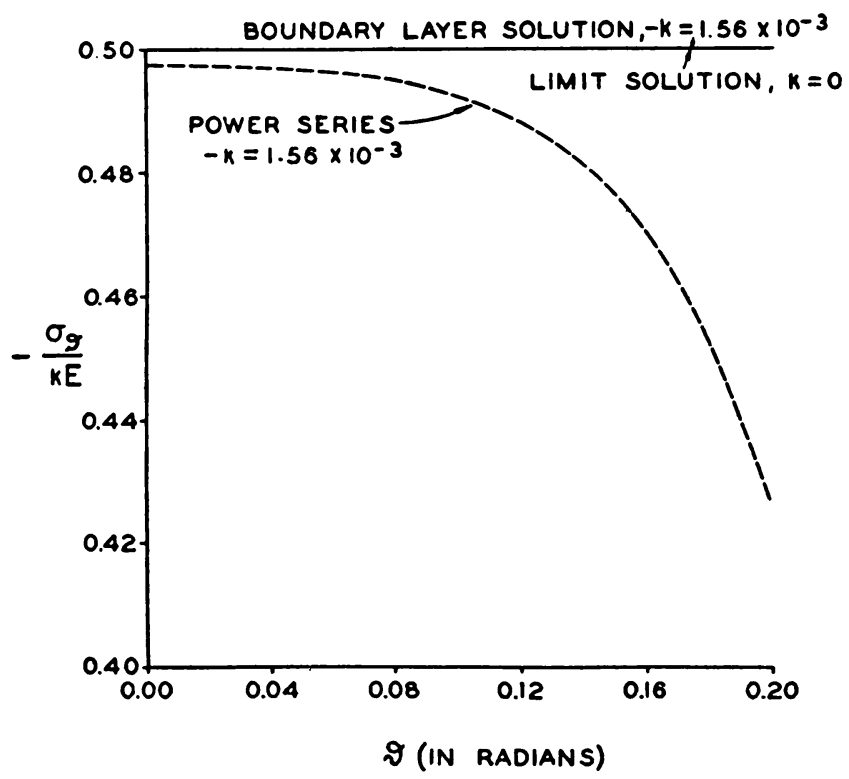

FIG. 5. Radial stress.

${ }^{16}$ The solution was obtained in the form of a development in powers of the independent variable $\theta$. Only four terms in the series (which appear not to converge very rapidly) were retained in calculating coefficients. On the graphs these solutions are marked "power series, $-\kappa=1.56 \times 10^{-3}$." 
for the case in which the value $\theta_{0}$ of $\theta$ at the edge of the sheet is 0.2 rad., and the dimensionless parameter ${ }^{17} \kappa=p R / E h$ has the value $-1.56 \times 10^{-3}$, and $\nu=0.3$. (This was one of the cases treated as part of the project mentioned in the footnote at the beginning of the paper.) The strain everywhere in the sheet is about $-\frac{1}{2}(1-\nu) \kappa$ in value. The graphs show values of $w / \kappa R,-\sigma_{\phi} / \kappa E$, and $-\sigma_{\theta} / \kappa E$ as functions of $\theta$. Each graph contains three curves: a dotted curve giving the result of our approximate solution; and two solid curves which refer to the limit solution obtained as $k \rightarrow 0$. The method of obtaining the limit solutions is explained in the next section. One observes that the curves marked "boundary layer solution" approximate those of our numerical solution rather well, at least for the stresses. We have some reason to think that a more accurate solution of the boundary value problem would show the boundary layer solution to be a better approximation to the actual solution than our graphs indicate. We note that the curves marked "limit solution, $\kappa=0$ " are those which would be obtained from the linear sheet theory.

7. The boundary layer problem. A boundary layer effect has already been mentioned a number of times in connection with our boundary value problem. The graphs of the solutions in the preceding section furnish a hint regarding the character of this phenomenon. The solutions in the interior portion of the sheet appear to be relatively constant, approaching there the values furnished by the linearized theory (i.e., those of the theory usually called the membrane theory of shells). However, toward the edge of the sheet, the solutions appear to change rather abruptly. This is consistent with the repeatedly mentioned fact that the condition for a fixed edge cannot be satisfied in the linearized theory. The purpose of the present section is to treat this boundary layer effect explicitly.

A necessary step in any treatment of boundary layer phenomena ${ }^{18}$ consists in the introduction of appropriate new variables and parameters. In the present case it is convenient to introduce new dimensionless dependent variables replacing $\epsilon_{\theta}, \epsilon_{\phi}, \sigma_{\theta}, \sigma_{\phi}$, $w$ and $u$ by the relations

$$
\begin{aligned}
s_{\theta} & =\sigma_{\theta} / E_{\kappa}, & s_{\phi} & =\sigma_{\phi} / E_{\kappa}, \\
\epsilon_{\theta} & =\epsilon_{\theta} / \kappa, & e_{\phi} & =\epsilon_{\phi} / \kappa, \\
\omega & =w / R_{\kappa}, & \mu & =u / R_{\kappa},
\end{aligned}
$$

in which the important dimensionless parameter $\kappa$ is defined by the relation

$$
\kappa=p R / E h \text {. }
$$

We assume here that the applied pressure $p$ is constant. In terms of the new quantities, the fundamental differential equations $(4.1),(4.2),(2.7)$, and (2.8) become, in order

$$
\begin{gathered}
\frac{d}{d \theta}\left(s_{\theta} \sin \theta\right)=\kappa\left(\frac{d \omega}{d \theta}+\mu\right) s_{\theta} \sin \theta+s_{\phi} \cos \theta, \\
\kappa \frac{d}{d \theta}\left[s_{\theta}\left(\frac{d \omega}{d \theta}+\mu\right) \sin \theta\right]=-\left[1+s_{\theta}+s_{\phi}\right] \sin \theta,
\end{gathered}
$$

${ }^{17}$ In the next section it will be seen that $\theta_{0}$ and $\kappa$ are the only essential parameters, once the value of the Poisson ratio $\nu$ is fixed.

${ }^{18}$ Such boundary layer effects have been well-known for many years in fluid mechanics. They occur also in problems in elasticity other than those considered in this paper. (See, for example $[7,8]$.) 


$$
\begin{aligned}
& e_{\theta}=\left(\frac{d \mu}{d \theta}-\omega\right)+\frac{\kappa}{2}\left(\frac{d \omega}{d \theta}+\mu\right)^{2}, \\
& e_{\phi}=(\mu \cot \theta-\omega) .
\end{aligned}
$$

To obtain a complete system of equations we add the stress-strain relations:

$$
\begin{aligned}
& e_{\theta}=s_{\theta}-\nu s_{\phi}, \\
& e_{\phi}=s_{\phi}-\nu s_{\theta} .
\end{aligned}
$$

As boundary conditions we require all quantities to be regular at $\theta=0$, while at $\theta=\theta_{0}$ the condition of a fixed edge is prescribed,

$$
\omega=0, \quad(7.11) \quad s_{\theta}-\nu s_{\phi}=0 .
$$

We now observe that if $\kappa$ is allowed to approach zero in these differential equations, the result is a set of differential equations for the limit quantities which are identically the same as those of the linear sheet theory ${ }^{19}$ (when formulated in terms of our dimensionless variables),

$$
\begin{gathered}
\frac{d}{d \theta}\left(s_{\theta} \sin \theta\right)=s_{\phi} \cos \theta, \\
e_{\theta}=\frac{d \mu}{d \theta}-\omega,
\end{gathered}
$$

$$
\begin{gathered}
s_{\theta}+s_{\phi}=-1, \\
e_{\phi}=\mu \cot \theta-\omega .
\end{gathered}
$$

Obviously, the boundary conditions (7.11) and (7.12) cannot be imposed in this limit problem. In fact, the solutions of (7.13) and (7.14) are completely determined by the regularity conditions at $\theta=0$ alone. This solution is, as we know, $s_{\theta}=s_{\phi}=-\frac{1}{2}$, $\omega=\frac{1}{2}(1-\nu), \mu=0$. In the limit, therefore, the boundary conditions at the edge, in general, will not be satisfied. It follows that the solution of the boundary value problem formulated in (7.5) to (7.12) will not converge uniformly at the boundary to the solution of the limit problem as $\kappa \rightarrow 0$, and this is the essential characteristic of a boundary layer effect.

It is, however, reasonable to expect that the solutions do converge in the interior (i.e., for $0 \leqq \theta_{\text {. }} \leqq \theta_{0}^{\prime}<\theta_{0}$, where $\theta_{0}^{\prime}$ is a constant) as $\kappa \rightarrow 0$ to the solutions of the limit problem for $\kappa=0$. The graphs of the preceding section confirm this to some extent.

It is possible to give an explicit treatment of the boundary layer effect. Such a treatment can be obtained through the introduction of a new independent variable which replaces $\theta$ and which depends on $\kappa$ in such a way that the solutions are made to converge uniformly at the boundary in the limit as $k \rightarrow 0$. What one wants, roughly speaking, is to stretch the boundary layer as $\kappa \rightarrow 0$ in such a way that its width does not shrink to zero. In our case, this can be accomplished by introducing as a new independent variable the quantity $\eta$ defined by the relation

$$
\eta=\frac{1}{\sqrt{-\kappa}}\left(\theta-\theta_{0}\right)
$$

19 It is perhaps of interest to observe that the limit situation characterized by $\kappa \rightarrow 0$ can be achieved by allowing the pressure $p$ to approach zero. However, if $p$ is simply set equal to zero in the original differential equations (4.1) and (4.2), the order of the system is not decreased. Thus the introduction of new dependent variables through division of the original ones by $\kappa$ is an essential step in the treatment of the boundary layer effect. 
The reason for the minus sign under the radical is that we wish to consider only cases for which the solutions are stable, which means cases in which $\kappa$ is negative. (Cf. the remarks at the end of Section 3.)

If we introduce the new independent variable in Eqs. (7.5) to (7.8) and then allow $\kappa$ to tend to zero, we obtain the set of limit differential equations

$$
\begin{array}{lll}
\frac{d s_{\theta}}{d \eta}=0, & (7.18) & \frac{d}{d \eta}\left(s_{\theta} \frac{d \omega}{d \eta}\right)=\left(1+s_{\theta}+s_{\phi}\right), \\
0=\frac{d \mu}{d \eta}, & (7.20) & e_{\phi}=\mu \cot \theta_{0}-\omega=s_{\phi}-\nu s_{\theta},
\end{array}
$$

for the range $-\infty<\eta \leqq 0$, where $\eta=0$ corresponds to the edge of the sheet. This system of equations, which has the same order as the original system, yields the boundary layer "resolution" which we seek. The boundary conditions at $\eta=0$ are given by (7.11) and (7.12). At $\eta=-\infty$ we expect all quantities to tend to the values furnished by the solution of the interior limit problem given above. Thus we expect $\omega$ to approach the value $\frac{1}{2}(1-\nu)$ as $\eta \rightarrow-\infty$.

Since the boundary layer differential equations have constant coefficients, they are readily solved by exponentials. One finds, for example, that $\omega$ satisfies the differential equation

$$
\frac{d^{2} \omega}{d \eta^{2}}-2 \omega=-1+\nu
$$

so that the homogeneous equation is solved by real exponentials. ${ }^{20}$ The solution of (7.22) which satisfies the conditions at $\eta=0$ and $\eta=-\infty$ is

$$
\omega=\frac{1}{2}(1-\nu)\left(e^{\sqrt{2}},-1\right) \text {. }
$$

The results for the other quantities are easily found to be

$$
\begin{gathered}
\mu \equiv 0, \quad(7.24) \quad s_{\theta} \equiv-\frac{1}{2}, \\
s_{\phi}=-\frac{1}{2}\left\{1+(1-\nu)\left(e^{\sqrt{2} \eta}-1\right)\right\} .
\end{gathered}
$$

The graphs of Figs. 3, 4, and 5 contain in each case a curve marked "boundary layer solution, $\kappa=-1.56 \times 10^{-3}$." These curves were obtained from (7.23), (7.25), and (7.26) by reintroduction of $\theta$ as a variable through use of (7.17) with $\kappa=-1.56 \times 10^{-3}$. Comparison with the curves for the numerical solution of the original boundary value problem indicates that such a "compressed" boundary layer solution may furnish a fairly good approximation to the values of $w$ and $\sigma_{\theta}$ near the edge of the sheet if $\kappa$ is not too large.

\section{REFERENCES}

[1] C. B. BIEzeno, Über die Bestimmung der Durchschlagkraft einer schwachgekrümmten, kreisförmigen Platte, Z. angew. Math. Mech. 15, 10-22 (1935).

[2] D. G. Bourgin, The clamped square sheet, Amer. J. Math. 16, 417-439 (1939).

[3] W. Z. ChIEn, The intrinsic theory of thin shells and plates, Parts I, II, III, Quart. Appl. Math. 1, 297-327 (1944); 2, 43-59, 120-135 (1944).

20 If we were to try to resolve the boundary layer in the case when $\kappa$ is positive (that is, the unstable case) the corresponding equation for $\omega$ would possess simple harmonic solutions and no limit would exist as $\eta \rightarrow-\infty$. 
[4] B. Eck, Z. angew. Math. Mech. 7, 498-500 (1927).

[5] W. FlüGge, Statik und Dynamik der Schalen, Julius Springer, Berlin, 1934.

[6] A. FöpPL, Vorlesungen über technische Mechanik, vol. 5, G. Teubner, Leipzig, 1907, §24.

[7] K. O. FRIEDRICHS, On the minimum buckling load for spherical shells, Applied Mechanics, Theodore von Kármán Anniversary Volume, 1941, pp. 258-272.

[8] K. O. FRIEDRICHS and J. J. STOKER, The non-linear boundary value problem of the buckled plate, Amer. J. Math. 63, 839-888 (1941). (1915).

[9] H. Hencky, Über den Spannungszustand in kreisrunden Platten, Z. Math. Phys. 63, 311-317 (1921).

[10] - Die Berechnung dünner rechteckiger Platten, Z. angew. Math. Mech. 1, 81-89, 423-424 (1910).

[11] T. von KÁRMÁN, Festigkeitsproblem im Maschinenbau, Encyk. d. math. Wiss. IV4, 311-385

[12] S. Timoshenko, Theory of plates and shells, McGraw-Hill, New York, 1940. 